\title{
KAKUTANI'S THEOREM FOR REAL-VALUED MAPS ON A COMPACT SURFACE
}

\author{
E. H. KRONHEIMER AND P. B. KRONHEIMER
}

\begin{abstract}
Let $M$ be a compact 2-manifold (without boundary) $C^{1}$-embedded in $\mathbf{R}^{3}$. Then there exists positive $\sigma$ such that, given any positive $\tau \leq \sigma$ and any continuous map $f: M \rightarrow \mathbf{R}$, there exist points $p, q, r \in M$, satisfying $\|q-r\|=\|r-p\|=\|p-q\|=\tau$ in the euclidean norm, for which $f(p)=$ $f(q)=f(r)$.
\end{abstract}

1. Introduction. A classical result known as Kakutani's theorem [5, 10] states that, given a real-valued map $f$ on the unit 2-sphere in the euclidean space $\mathbf{R}^{3}$, there exists an equilateral triangle in $\mathbf{R}^{3}$, of any specified side-length not exceeding $\sqrt{3}$, whose vertices lie on a contour of $f$. In 1955 Floyd [4] proved the corresponding proposition for nonequilateral triangles; while successive stages of the generalization of the theorem to regular $n$-simplexes and maps on the euclidean $n$-sphere were proved, between 1950 and 1969, by Yamabe and Yujobô [11], Bourgin [1] and Fenn [2]. The $n$-dimensional counterpart of Floyd's result was conjectured as early as 1947 by Knaster [6] but remains unproved.

All the proofs listed above are global. In this note we present a strongly geometric, but local, proof of Kakutani's theorem, to establish the perhaps surprising result that it holds on any compact 2-manifold smoothly embedded in $\mathbf{R}^{3}$. An elementary example, such as the map $f(x, y, z)=x$ on the surface $x^{2}+y^{2}+(10 z)^{2}=1$, is enough to show, however, that in the general case it is no longer possible to take the size of the largest equilateral triangle whose vertices can be inscribed on the manifold as the upper limit on the size of admissible triangles.

\section{Statements and proofs.}

THEOREM 1. Let $M$ be a compact 2-dimensional $C^{1}$ submanifold of $\mathbf{R}^{3}$. Then there exists $\sigma>0$ such that, for each continuous real-valued map $f$ on $M$ and each positive number $\tau \leq \sigma$, the space $\mathbf{R}^{3}$ contains an equilateral triangle of side-length $\tau$ whose vertices $p, q, r$ lie on $M$ and satisfy $f(p)=f(q)=f(r)$.

We begin the proof of Theorem 1 by constructing a (not necessarily maximal) value for $\sigma$.

For each point $p$ of $M$ we denote by $T_{p}$ the affine plane in $\mathbf{R}^{3}$ tangent to $M$ at $p$ and by $n_{p}$ the unit vector in $\mathbf{R}^{3}$ normal to $T_{p}$ whose sense is consonant with specified orientations of $\mathbf{R}^{3}$ and of $M$. Let $\psi_{p}$ denote the orthogonal projection of $\mathbf{R}^{3}$ onto $T_{p}$; then the point $p$ has a neighbourhood $U_{p}$ in $M$ which is mapped by $\psi_{p}$ diffeomorphically into $T_{p}$. We choose $U_{p}$ so that (i) its image under $\psi_{p}$ is a closed circular disc $D_{p}$, centre $p$, whose radius we shall call $3 \sigma_{p}$, and (ii) the angle between

Received by the editors June 15, 1981.

1980 Mathematics Subject Classification. Primary 53A05; Secondary 55M20. 
$n_{p}$ and $n_{q}$ is less than $\pi / 8$ at every point $q$ of $U_{p}$. Let $E_{p}$ and $F_{p}$ be the closed circular discs in $T_{p}$, with centre $p$, whose radii are respectively $2 \sigma_{p}$ and $\sigma_{p}$; and let $V_{p}=U_{p} \cap \psi_{p}^{-1}\left(E_{p}\right)$ and $W_{p}=U_{p} \cap \psi_{p}^{-1}\left(F_{p}\right)$. Let $M^{\prime}$ be a finite subset of $M$ such that $\left\{W_{q}\right\}_{q \in M^{\prime}}$ covers $M$, and define $\sigma=\min \left\{\sigma_{q}\right\}_{q \in M^{\prime}}$.

Let $\tau$ be a number satisfying $0<\tau \leq \sigma$, let $f$ be a continuous real-valued map on $M$, and let $c$ be a point at which $f$ attains its minimum value. We can assume that $f(c)=0$, so that $f$ is nowhere negative. Let $o$ be a point of $M^{\prime}$ such that $c \in W_{o}$. Since we shall be restricting our attention to the vicinity of $o$, it will be convenient to omit the suffix $o$ from the symbols $D_{o}, E_{o}, F_{o}, U_{o}, V_{o}, W_{o}, n_{o}$ and $\psi_{0}$

LEMMA 1. If $p, q$ are distinct points of $U$, then the vector $p-q$ makes an angle greater than $3 \pi / 8$ with $n$. In particular, therefore, $(p-q) \cdot n<\frac{1}{2}\|p-q\|$.

Proof. This is merely the increment formula for the $C^{1} \operatorname{map} s \mapsto n \cdot\left(s^{*}-s\right)$ on the line segment $[\psi(p), \psi(q)]$, where $\left\{s^{*}\right\}=U \cap \psi^{-1}(s)$.

It follows from Lemma 1 that no equilateral triangle with all its vertices in $U$ lies in a plane parallel to $n$, and that consequently, if $\Omega$ denotes the specified orientation of $\mathbf{R}^{3}$, then the set of triangles

$$
\tau=\left\{(p, q, r) \in U^{3}\|\| q-r\|=\| r-p\|=\| p-q \|=\tau ;(n, p-r, q-r) \in \Omega\right\},
$$

regarded as a subspace of $\left(\mathbf{R}^{3}\right)^{3}$, is compact. We shall write $\alpha$ for the projection from $\tau$ to $U$ which maps the triangle $(p, q, r)$ to its first vertex $p$ and let $\mathcal{V}$ and $W$ denote the subspaces $\alpha^{-1}(V)$ and $\alpha^{-1}(W)$ of $\tau$.

For any two distinct points $p$ and $q$ in $U$ we write

$$
w(p, q)=(\psi(p)-\psi(q)) /\|\psi(p)-\psi(q)\|
$$

and we define

$$
\Theta: \mathcal{V} \rightarrow V \times S^{1}: \Delta \mapsto(\alpha(\Delta), \omega(\Delta))
$$

where $S^{1}$ denotes the space of unit vectors in $\mathbf{R}^{3}$ orthogonal to $n$, and $\omega(p, q, r)=$ $w(p, q)$.

LEMMA 2. The map $\Theta$ is a homeomorphism.

ProOF. Since $\theta$ is certainly continuous and $\mathcal{V}$ is compact, we have only to establish that $\boldsymbol{\theta}$ is invertible. We prove injectivity first.

Let $p, q, q^{\prime}$ be points of $U$ such that $\|p-q\|=\left\|p-q^{\prime}\right\|=\tau$ and $w(p, q)=$ $w\left(p, q^{\prime}\right)$. Then $q$ and $q^{\prime}$ lie on a semicircular arc with endpoints $p \pm \tau n$. By Lemma 1 applied to the pairs $p, q$ and $p, q^{\prime}$, however, both $|(p-q) \cdot n|$ and $\left|\left(p-q^{\prime}\right) . n\right|$ are less than $\frac{1}{2} \tau$; and this is easily seen to contradict Lemma 1 applied to the pair $q, q^{\prime}$ unless $q=q^{\prime}$.

Now suppose that $\mathcal{V}$ contains two distinct triangles $\left(p, q, r_{1}\right)$ and $\left(p, q, r_{2}\right)$. Let $L$ be the circle $\{r \mid\|p-r\|=\|q-r\|=\tau\}$, and let $L$ intersect the plane through $p$ and $q$ parallel to $n$-unique by Lemma 1 -in the points $l$ and $l^{\prime}$. Then $L \backslash\left\{l, l^{\prime}\right\}=$ $L^{+} \cup L^{-}$, where $L^{+}, L^{-}$are two semicircular arcs such that

$$
r \in L^{ \pm} \Leftrightarrow(n, p-r, q-r) \in \pm \Omega
$$

and $r_{1}, r_{2} \in L^{+}$. The orthogonal vectors $l-l^{\prime}$ and $p-q$ are coplanar with $n$; so $l-l^{\prime}$ must make an angle less than $\pi / 8$ with either $n$ or $-n$. Put $m$ for the centre $\frac{1}{2}(p+q)$ of $L$; then, by Lemma $1,(r-m) \cdot n<\frac{1}{2} \tau$ whenever $(p, q, r) \in \mathcal{V}$. It follows 
that $r_{1}, r_{2}$ both lie in the arc cut off on $L^{+}$by the two lines in the plane of $L$ which are orthogonal to the vector $l-l^{\prime}$ and at distance $\frac{1}{2} \tau \sec (\pi / 8)$ from $m$. But this implies that $l-l^{\prime}$ makes an angle less than $\sin ^{-1}((1 / \sqrt{3}) \sec (\pi / 8))(<\pi / 4)$ with either $r_{1}-r_{2}$ or $r_{2}-r_{1}$. Hence either $r_{1}-r_{2}$ or $r_{2}-r_{1}$ must make an angle less than $(\pi / 8)+(\pi / 4)$ with $n$, which contradicts Lemma 1 .

To prove surjectivity, we choose $p \in V$ and $k \in S^{1}$ and show there exist $q$ and $r$ such that $w(p, q)=k$ and $(p, q, r) \in \tau$. The existence of a point $q$ in $U$ satisfying $w(p, q)=k$ and $\|p-q\|=\tau$ follows from the observation that (since $\psi(p) \in E$ and $\tau \leq \sigma)$

$$
\{\psi(p)+\lambda k \mid 0 \leq \lambda \leq \tau\} \subset D
$$

For a pair of points $p$ and $q$ so specified, we define $l, l^{\prime}$ and $L^{+}$as before. The complement of $U$ in the cylinder $X=U+n \mathbf{R}$ has two components, $X^{ \pm}=U+$ $n \mathbf{R}^{ \pm}$. The semicircular arc $L^{+} \cup\left\{l, l^{\prime}\right\}$ lies wholly in $X$; and-by Lemma 1 -one of its end-points is in $X^{+}$and the other in $X^{-}$. The arc must therefore intersect $U$ : and a point of intersection is the required $r$.

We shall write $\rho(p, q, r)=(q, r, p)$; so that both $\rho$ and $\rho^{2}$ map $\mathcal{W}$ into $\mathcal{V}$. We let $\mathbf{C}$ denote the unit circle in the complex plane and put $u=e^{2 \pi i / 3}$. Recall that $c$ is a zero of $f$ in $W$.

LEMMA 3. There exist homotopic maps $g, h: \mathbf{C} \rightarrow \mathcal{V}$ such that $g(u z)=\rho(g(z))$ and $\alpha(h(z))=c$ for all $z \in \mathbf{C}$.

Proof. By Lemma 2, there exists $\Delta \in \mathcal{V}$ satisfying $\alpha(\Delta)=o$, which implies that $\Delta \in W^{3}$. Both $\Delta$ and $\rho(\Delta)$ are consequently in the space $W$; and this-by another application of Lemma 2-is homeomorphic to $W \times S^{1}$ and therefore pathconnected. Let $\chi:[0,1] \rightarrow W$ be a path from $\Delta$ to $\rho(\Delta)$. We define $g$ by putting

$$
g\left(e^{2 \pi i \lambda}\right)=\rho^{\nu}(\chi(3 \lambda-\nu)) \quad(\nu \leq 3 \lambda \leq \nu+1)
$$

for $\nu=0,1,2$, and $h$ by putting

$$
h(z)=\boldsymbol{\Theta}^{-1}(c, \omega(g(z))) \quad(z \in \mathbf{C}),
$$

so that $\omega \circ g=\omega \circ h$. Since $V$ is contractible, $\alpha \circ g \simeq \alpha \circ h$. It follows that $\Theta \circ g \simeq \Theta \circ h$ and hence (by Lemma 2) that $g \simeq h$.

Suppose now that for no triangle $(p, q, r)$ in $\mathcal{V}$ does $f(p)=f(q)=f(r)$. Then two homotopic maps $G, H: \mathbf{C} \rightarrow \mathbf{C}$ can be defined by putting $G=\xi \circ g$ and $H=$ $\xi \circ h$, where

$$
\xi(p, q, r)=\left(f(p)+u f(q)+u^{2} f(r)\right) /\left|f(p)+u f(q)+u^{2} f(r)\right|
$$

and $g, h$ have the properties specified in the statement of Lemma 3. Since $f$ is nonnegative and vanishes at $c$, the image of $H$ lies in the arc $\left\{e^{2 \pi i \lambda} \mid 1 \leq 3 \lambda \leq 2\right\}$; so $H$ is null-homotopic. On the other hand, since $\xi(\rho(\Delta))=u^{2} \xi(\Delta)$ for every $\Delta \epsilon$ $\mathcal{V}$, we have $G(u z)=u^{2} G(z)$ for every $z \in \mathbf{C}$; so the map $G^{\uparrow}: \mathbf{C} \rightarrow \mathbf{C}$ defined by $G^{\dagger}(z)=z G(z)$ satisfies $G^{\dagger}(u z)=G^{\dagger}(z)$, which implies that degree $G^{\dagger}$ is a multiple of 3. But degree $G^{\uparrow}=1+$ degree $G=1+$ degree $H=1$. This contradiction completes the proof of Theorem 1 .

Note that we have used only the local structure of the manifold $M$ : in particular no essential use has been made of its global orientability. There is consequently no difficulty in re-formulating the argument to establish the following stronger result. 
THEOREM 2. Let $i: M \rightarrow \mathbf{R}^{3}$ be a $C^{1}$ immersion of a compact 2-manifold. Then there exists $\sigma>0$ such that, for each real-valued continuous map $f$ on $M$ and each positive number $\tau \leq \sigma$, there are points $p, q, r$ in $M$ satisfying

$$
\|i(q)-i(r)\|=\|i(r)-i(p)\|=\|i(p)-i(q)\|=\tau
$$

for which $f(p)=f(q)=f(r)$.

3. Observations. Apart from the obvious question of whether these results admit the same generalizations as Kakutani's theorem, it is reasonable to ask whether they do not remain valid even if $M$ is only topologically embedded or immersed in $\mathbf{R}^{3}$. Already the 1-dimensional case, however, seems difficult to handle without some assumption of smoothness; though a much-simplified version of the preceding argument establishes the following counterpart to Theorem 2.

THEOREM 3. Let $\Gamma: \mathbf{C} \rightarrow \mathbf{R}^{2}$ be a $C^{1}$ immersion. Then there exists $\sigma>0$ such that, for each continuous real-valued map $f$ on $\mathbf{C}$ and each positive number $\tau \leq \sigma$, there are points $p, q$ in $\mathbf{C}$ such that $\|\Gamma(p)-\Gamma(q)\|=\tau$ and $f(p)=f(q)$.

That the same proposition holds if the curve $\Gamma$ is a piecewise analytic embedding follows from a result due to Fenn [3]. Fenn showed that, taking $\sigma$ to be the (necessarily positive) Livesay width of the curve $[9,8]$, it is possible to slide a chord of any length $\tau \leq \sigma$ around the curve-in the sense that there exist two maps $F_{1}, F_{2}: \mathbf{C} \rightarrow \mathbf{C}$ of degree 1 such that $\left\|\Gamma\left(F_{1}(z)\right)-\Gamma\left(F_{2}(z)\right)\right\|=\tau$ for all $z$-which implies the conclusion of Theorem 3 .

A somewhat different question is whether these results are true for a compact 2manifold-with-boundary embedded in $\mathbf{R}^{3}$, provided we add the restriction (without which they are clearly false) that the map $f$ should vanish at every boundary point. With this restriction Theorem 1 is known to hold for any compact convex disc [7]. The corresponding 1-dimensional result, on the other hand, fails irremediably.

Proposition. A necessary and sufficient condition that for every continuous map $f:[0,1] \rightarrow \mathbf{R}$ satisfying $f(0)=f(1)=0$ there should exist points $p, q \in[0,1]$ satisfying $|p-q|=\tau$ and $f(p)=f(q)$, is that $\tau \in\{0,1,1 / 2,1 / 3, \ldots\}$.

PROOF. If $\tau^{-1}$ is an integer and $f$ is given, choose an integer $k$ which maximizes $|f(k \tau)|$. Then $f(k \tau-\tau)-f(k \tau)$ and $f(k \tau)-f(k \tau+\tau)$ are of opposite sign (or zero); and the existence of $p$ and $q$ follows. If $\tau^{-1}$ is not an integer, consider the map

$$
f(x)=\left(1-\cos 2 \pi \tau^{-1}\right) x-\left(1-\cos 2 \pi \tau^{-1} x\right) .
$$

\section{REFERENCES}

1. D. G. Bourgin, Deformations and mapping-theorems, Fund. Math. 46 (1959), 285-303.

2. R. A. Fenn, A survey of and some original contributions to theorems of the Borsuk-Ulam-KakutaniYamabe-Yujobô type, Ph. D. thesis, Univ. of London, 1969.

3. $\ldots$ Sliding a chord and the width and breadth of a closed curve, J. London Math. Soc. (2) 5 (1972), 39-47.

4. E. E. Floyd, Real-valued mappings of spheres, Proc. Amer. Math. Soc. 6 (1955), 957-959.

5. S. Kakutani, A proof that there exists a circumscribing cube around any bounded closed convex set in $\mathbf{R}^{3}$, Ann. of Math. 43 (1942), 739-741.

6. B. Knaster, Problem 4, Colloq. Math. 1 (1947), 30-31.

7. E. H. Kronheimer and P. B. Kronheimer, The Tripos problem, J. London Math. Soc. (2) 24 (1981), 182-192. 
8. D. G. Larman, On a conjecture of Roger Fenn for the width and breadth of a Jordan curve, Proc. London Math. Soc. (3) 28 (1974), 159-173.

9. G. R. Livesay, Concerning real-valued maps of the n-sphere, Proc. Amer. Math. Soc. 8 (1957), 989-991.

10. A. de Mira Fernandes, Funvioni continue sopra una superficie sferica, Portugal. Math. 5 (1946), 132-134.

11. H. Yamabe and Z. Yujobô, On the continuous functions defined on a sphere, Osaka Math. J. 2 (1950), 19-22.

Department of Mathematics, Birkbeck College, Malet Street, London WC1E 7HX, ENGLAND

Merton COLlege, OXFord OX1 4JD, ENGLAND 